\title{
Severe Self-Inflicted Acute Ocular Siderosis Caused by an Iron Tablet in the Conjunctival Fornix
}

\author{
Ioannis Asproudis*, Paraskevas Zafeiropoulos, Andreas Katsanos, Christos Kolettis
}

\begin{abstract}
We present the case of a female patient who grinded a ferrous sulfate tablet and placed it at the conjunctival fornix of her left eye. She rapidly developed severe ocular siderosis, with profoundly decreased visual acuity, corneal opacities, cataract, retinal degeneration and ultimately phthisis bulbi. To our knowledge, this is the first report on the consequences of application of an iron tablet on the conjunctiva.
\end{abstract}

\section{KEYWORDS}

siderosis; siderosis bulbi; self-inflicted injury; phthisis; oedipism; deferoxamine

\section{AUTHOR AFFILIATIONS}

Ophthalmology Clinic of the Medical School of the University of Ioannina, loannina, Greece

* Corresponding author: Stavros Niarchos Avenue, Ophthalmology Clinic, University Hospital, 45500 loannina, Greece; e-mail: asproudisi@yahoo.gr

Received: 13 August 2017

Accepted: 25 January 2018

Published online: 23 April 2018

Acta Medica (Hradec Králové) 2017; 60(4): 160-162

https://doi.org/10.14712/18059694.2018.13

(c) 2017 The Authors. This is an open-access article distributed under the terms of the Creative Commons Attribution License (http://creativecommons.org/licenses/by/4.0), which permits unrestricted use, distribution, and reproduction in any medium, provided the original author and source are credited. 


\section{CASE REPORT}

A 45-years old white female patient presented to the emergency service claiming she had injured her left eye when she accidentally fell the previous day. Her general medical history was only significant for schizophrenia under medication. The patient appeared well oriented in time, place and person and behaved appropriately in all occasions.

Snellen best corrected visual acuity (VA) was 10/10 in her right eye (OD) and light perception in her left eye (OS). Intraocular pressure was $13 \mathrm{mmHg}$ in OD, but could not be measured in OS due to hypotony. The examination of OD was unremarkable. The OS had excessive palpebral edema, chemosis and a dark brown material mostly along the temporal part of the limbus. This material was initially thought to be part of the ciliary body or iris. The cornea was edematous with an unusual brown hue, whitish stromal infiltrates, epithelial erosions and brownish endothelial precipitates. Fibrinous material was noted in the anterior chamber. The crystalline lens was diffusely yellow with cortical and anterior subcapsular opacities. Fundoscopy was not possible. Computed tomography of the orbits revealed a poorly defined radiopaque area with-

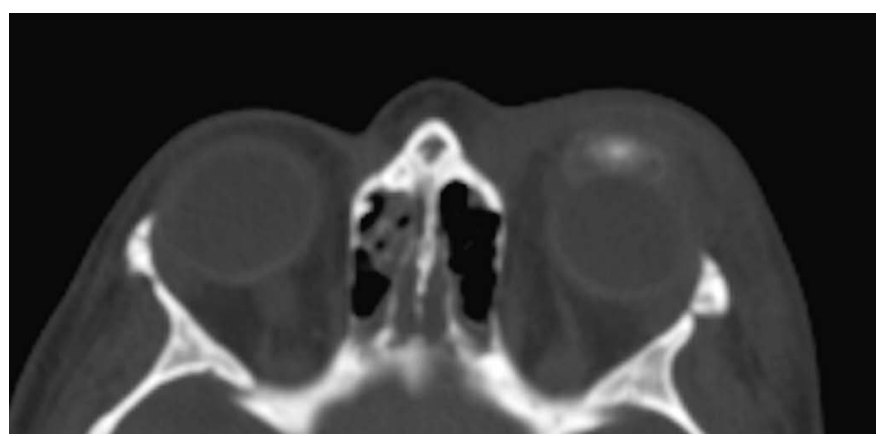

Fig. 1 Poorly-circumscribed radiopaque area on computed tomography scan in left eye. Edema of the peribulbar soft tissues is noted.

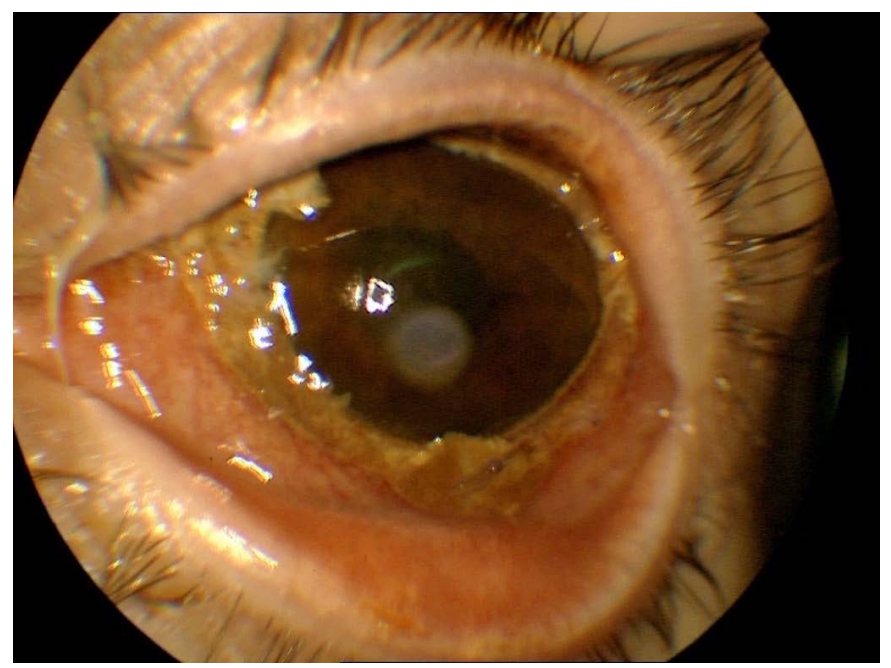

Fig. 2 Slit lamp photograph of the patient's left eye after surgical exploration and debridement. Chemosis, hyperaemia and corneal infiltration are shown. Residual necrotic conjunctival tissue appears brown. in the structures of the anterior segment in OS (Fig. 1). Surgical exploration revealed no site of injury or foreign body. Large areas of necrotic, brown, frail conjunctiva and sclera were identified, especially underneath the superior eyelid. Careful dissection of the necrotic tissue was performed (Fig. 2).

On further questioning, the patient eventually admitted she had grinded a single ferrous sulfate tablet and placed the powder at the superior conjunctival fornix of her left eye. Self-inflicted ocular siderosis was diagnosed and urgent psychiatric evaluation was arranged. The patient was started on cefuroxime intravenously, methylprednisolone per os, atropine eyedrops b.i.d., chloramphenicol-dexamethasone eyedrops t.i.d. and deferoxamine mesylate $10 \%$ eyedrops q.i.d. After several days of in-patient treatment the VA in OS was still light perception, the anterior chamber was shallow with fibrinous material and the eye remained hypotonous. Phthisis ensued within the next couple of weeks.

\section{DISCUSSION}

To the best of our knowledge, there has been no previous report of self-inflicted ocular siderosis using iron tablets. Ocular self-mutilation has been described in schizophrenia, drug-induced psychosis, manic phase of bipolar disorder, depression, and in certain organic diseases (Lesch-Nyhan syndrome, delirium tremens, encephalitis) (1). The different forms of self-inflicted injuries include blunt trauma, penetrating wounds, and unilateral or bilateral enucleation (oedipism) (1).

Ocular siderosis is an uncommon, potentially blinding condition usually caused by an iron-containing retained intraocular foreign body (IOFB) $(2,3)$. Cell death results from the toxic effect of iron on cellular enzyme systems $(2,4)$. The condition may appear from few days up to many years following the injury and any ocular tissue from the cornea to the optic nerve may be affected $(2,3)$. The clinical severity depends on the size and shape of the IOFB, its iron content and the time it remains in the eye. The most common manifestations are cataract formation (especially anterior subcapsular), diffuse pigmentary changes of the retinal pigment epithelium, iris heterochromia often in conjunction with a tonically dilated pupil, secondary glaucoma, iritis and cystoid macular edema (4). Retinal arteriolar attenuation, proliferative vitreoretinopathy and retinal detachment have also been described $(2,3,4)$.

There are two reasons why our patient suffered a rapid and severe deterioration. Firstly, the quantity of iron in the tablet ( $256.3 \mathrm{mg}$ of iron sulfate corresponding to $80 \mathrm{mg}$ of elemental iron) is significantly larger than that of a typical IOFB. Secondly, the speed of iron release from a tablet is much faster than that of an IOFB: at least in the gastrointestinal tract, these tablets dissolve within approximately 7 hours.

The management of IOFB-induced ocular siderosis involves the removal of the foreign body $(2,4)$. In our case, when surgical exploration was undertaken, practically all ocular tissues were already saturated with iron as sug- 
gested by their brown colour and brittle texture (Fig. 2). Deferoxamine is a chelator with high iron affinity that has been used subconjunctivally or topically as $10 \%$ solution for the management of ocular siderosis (5). In our case, its use proved ineffective because irreversible tissue damage had already occurred at presentation.

Our case illustrates the challenges of dealing with self-inflicted ocular injuries in psychiatric patients. It further illustrates that upon contact with the eye, ferrous sulfate tablets can cause severe tissue necrosis that can rapidly culminate to irreversible blindness despite all efforts. Ophthalmologists should have a high index of suspicion when treating ocular injuries in psychiatric patients.
Prompt intervention and close collaboration with psychiatrists may reduce the risk of significant handicap.

\section{REFERENCES}

1. Patton N. Self-inflicted eye injuries: a review. Eye 2004; 18: 867-872.

2. Kannan NB, Adenuga OO, Rajan RP, Ramasamy K. Management of ocular siderosis: visual outcome and electroretinographic changes. J Ophthalmol 2016; 2016: 7272465

3. Lapira M, Karl D, Murgatroyd H. Siderosis bulbi as a consequence of a missed intraocular foreign body. BMJ Case Rep 2014 Jan 23; 2014

4. Sandhu HS, Young LH. Ocular siderosis. Int Ophthalmol Clin 2013; 53: $177-184$.

5. Valvo A, Del Duca A. Applications of a new chelating substance in ophthalmology. Therapeutic results in a case of ocular siderosis treated with desferrioxamine B. Ann Ottalmol Clin Ocul 1965; 91: 497-513. 